\title{
Uso do escore APACHE IV como preditor de mortalidade e tempo de permanência em uma unidade de terapia intensiva
}

\author{
Use of the APACHE IV score as a predictor of mortality and length \\ of stay in an intensive care unit
}

\author{
Gyzelly Alves de Carvalho', Adriana Arruda Barbosa Rezende², \\ Geovane Rossone Reis ${ }^{3}$, Giulliano Gardenghi ${ }^{4}$

\begin{abstract}
'Universidade de Gurupi. Gurupi, Tocantins, Brasil. ORCID: 0000-0002-1592-9870. gyzellya.c@gmail.com 2Universidade de Gurupi. Gurupi, Tocantins, Brasil. ORCID: 0000-0003-3642-3024. drikas.arruda@gmail.com ${ }^{3}$ Universidade de Gurupi. Gurupi, Tocantins, Brasil. ORCID: 0000-0002-6549-2371. georossone@hotmail.com
\end{abstract} \\ ${ }^{4}$ Autor para correspondência. Centro de Estudos Avançados e Formação Integrada (Goiânia), Hospital Encore (Aparecida de Goiânia). \\ Goiás, Brasil. ORCID: 0000-0002-8763-561X. ggardenghi@encore.com.br
}

RESUMO | INTRODUÇÃO: A avaliação do risco de morte e o tempo estimado de permanência em Unidade de Terapia Intensiva (UTI) é uma prática clínica relevante para predizer a gravidade da doença e traçar estratégias eficazes para a melhora do paciente e dos indicadores de qualidade do hospital. OBJETIVO: Avaliar a confiabilidade do escore APACHE IV como preditor de mortalidade e tempo de permanência em uma UTI do sul do estado do Tocantins. MATERIAL E MÉTODO: Pesquisa de caráter descritivo e quali-quantitativo realizada nos prontuários dos pacientes internados em uma UTI do sul do estado do Tocantins. Foram colhidas informaç̃os nos prontuários e aplicado o escore APACHE IV nos pacientes internados na UTI do sul do estado do Tocantins. no período de 24 de Outubro a 26 de Novembro de 2018. Foram excluídos os pacientes com período de internação inferior a 24 horas, que não realizaram todos os exames necessários para o APACHE IV, que foram transferidos do setor ou que não tiveram alta ou óbito ao final dessa pesquisa. Foi utilizado o coeficiente de correlação de Spearman para examinar a relação entre o escore APACHE-IV e o tempo de internação na UTI e para verificar a acurácia do APACHE-IV para mortalidade, à curva Receiver Operator Characteristic (ROC) com uma atribuição de 'bom'> 0,80. RESULTADOS: $O$ escore APACHE IV foi aplicado em dez pacientes, sendo que este superestimou o período de permanência dos pacientes internados na UTI em estudo, com $p<0,001$ e a mortalidade geral, com diferença absoluta de $20 \%(p=0,447)$. CONCLUSÃO: Baseada neste estudo, o APACHE IV não demonstrou confiabilidade para predição de mortalidade e tempo de permanência, porém a amostra insuficiente pode ter contribuído com esta conclusão.
ABSTRACT | INTRODUCTION: The assessment of the risk of death and the estimated length of stay in the intensive care unit (ICU) is a relevant clinical practice to predict the severity of the disease and to outline effective strategies for patient improvement and hospital quality indicators. OBJECTIVE: To evaluate the reliability of the APACHE IV score as a predictor of mortality and length of stay in a ICU in the southern state of Tocantins. MATERIAL AND METHOD: This is a descriptive and qualitative research conducted in the medical records of patients hospitalized in a suppressed ICU. Information was collected from the medical records and the APACHE IV score was applied to patients hospitalized in the ICU of the SUPRIMIDO from October 24 to November 26, 2018. Patients with hospitalization less than 24 hours who did not undergo all necessary examinations were excluded. for APACHE IV, who were transferred from the sector or who were not discharged or died at the end of this survey. Spearman's correlation coefficient was used to examine the relationship between the APACHE-IV score and ICU length of stay and to verify the accuracy of APACHE-IV for mortality, to the Receiver Operator Characteristic (ROC) curve with an assignment of ' good '> 0.80. RESULTS: The APACHE IV score was applied to ten patients, which overestimated the length of stay of ICU patients, with $p<0.001$ and overall mortality, with an absolute difference of $20 \%$. $(p=0.447)$. CONCLUSION: Based on this study, APACHE IV did not show reliability for predicting mortality and length of stay, but the insufficient sample may have contributed to this conclusion.

KEYWORDS: Intensive care unit. APACHE. Mortality.

PALAVRAS-CHAVE: Unidade de terapia intensiva. APACHE. Mortalidade. 


\section{Introdução}

A avaliação clínica é uma parte importante da prática médica em que prevê o prognóstico de pacientes admitidos em Unidades de Terapia Intensiva (UTI). Informações sobre as características do estado clínico podem ajudar na definição de estratégias, traçando planos na melhoria do atendimento, auxiliando na prevenção de complicações e atingindo melhores indicadores de qualidade. Os conhecimentos dessas características possibilitam aos profissionais de saúde o planejamento desses cuidados independente do agravo que motivou a internação'.

As tecnologias utilizadas em UTI incrementam a sobrevida de pacientes críticos, e em contrapartida aumentam os fatores de risco predisponentes que os levam a adquirir Infecções Relacionadas à Assistência a Saúde (IRAS), principalmente a pneumonia associada à ventilação mecânica (PAVM). Dentre os fatores de risco está o uso indiscriminado de antimicrobiano prévio, a gravidade da doença de base, tempo de permanência na UTI e os procedimentos invasivos a que são submetidos ${ }^{2}$.

Todos os pacientes admitidos em UTI são expostos a uma série de eventos que podem muitas vezes, resultar em um tempo de permanência prolongado ou, devido à gravidade do seu prognóstico podem acabar evoluindo a óbito. Internações por comprometimentos cardiovasculares e respiratórios têm um índice elevado no tempo de permanência e na mortalidade. Esses índices são avaliados por métodos altamente qualificados que pode predizer o tempo de permanência e a sobrevida desses pacientes ${ }^{3}$.

Um dos modelos mais conhecidos é o sistema de escore Acute Physiology And Chronic Healh Evaluation (APACHE- Avaliação do Estado de Saúde Agudo e Crônico) que avalia o tempo de permanência e a mortalidade em pacientes internados em UTI ${ }^{4}$. O escore APACHE teve sua primeira versão desenvolvida em 1981 em um estudo que considerou vários parâmetros incluindo sinais vitais, variáveis fisiológicas, pontuação neurológica, produção de urina, idade e condições mórbidas. Sua última versão foi introduzida em 2006, com 129 variáveis colhidas nas primeiras 24 horas de admissão. Dentre todos os métodos de avaliação, o APACHE IV talvez seja o mais utilizado devido a sua fácil aplicação e por basear-se em dados disponíveis na maior parte dos hospitais ${ }^{5}$.
Na prática clínica é importante um sistema confiável para categorizar os pacientes, identificando a gravidade da doença e a efetividade da terapêutica, a fim de contribuir na tomada de decisões e melhorar os indicadores de qualidade do hospital. Diante disso, este estudo teve por objetivo avaliar a confiabilidade do escore APACHE IV como preditor de mortalidade e tempo de permanência em uma UTI do sul do estado do Tocantins.

\section{Materiais e métodos}

Trata-se de uma pesquisa de caráter descritivo e quali-quantitativo realizada nos prontuários dos pacientes admitidos na UTI geral que possui dez leitos de um hospital do Sul do Estado do Tocantins entre 24 de outubro a 26 de novembro de 2018, após aprovação do Comitê de Ética em Pesquisa da Universidade de Gurupi - UNIRG sob o protocolo 2.930 (CAAE 97880918.4.0000.5518) e assinatura do Termo de Consentimento Livre e Esclarecido realizada pelos responsáveis dos pacientes admitidos na UTI. A escolha do período da coleta dos dados se deu pela maior possibilidade de admissões de novos pacientes, visto que o setor havia dado alta há $80 \%$ da capacidade de ocupação.

Todos os pacientes internados no período supracitado que atenderam os critérios de inclusão e exclusão fizeram parte da amostra. Os critérios de inclusão adotados foram indivíduos de ambos os sexos, de toda faixa etária (neonatal, pediátrico e adulto) e em diferentes especialidades. Foram excluídos os pacientes com período de internação inferior a 24 horas, que não realizaram todos os exames necessários para o APACHE IV, os que foram transferidos do setor ou que não tiveram alta ou óbito até o final desta pesquisa.

Alguns dados foram extraídos a partir da análise dos prontuários dos pacientes internados no período estabelecido. Foram levadas em conta as seguintes informações presentes: leito, número de prontuário, data e hora da internação, sexo, idade, unidade proveniente, diagnostico de internação, doenças associadas, data e hora da alta, óbito ou transferência, e destino no caso de transferência, nível de sedaçãoRASS (Richamond Agitation- Sedation Scale) e média dos dias de internação6 . 
O APACHE IV foi calculado nas primeiras 24 horas de internação pela coordenadora da fisioterapia responsável pelo setor. As variáveis utilizadas para calcular o escore APACHE-IV foram: idade, sexo, datas de admissão, alta ou óbito, pressão arterial sistólica e diastólica, temperatura corporal, frequência cardíaca, frequência respiratória, glicemia, uréia, soro, creatinina, hematócrito, glóbulos brancos, albumina sérica e bilirrubina, débito urinário durante as primeiras 24 horas de internação na UTI, ph, fração inspirada de oxigênio (FiO2), pressão parcial de dióxido de carbono ( $\mathrm{PaCO} 2)$, pressão parcial de oxigênio ( $\mathrm{PaO} 2)$, bicarbonato (HCO3), escala de coma de Glasgow, ventilação mecânica (VM) e diagnóstico da admissão7. A média da pressão arterial foi dada por duas aferições realizadas no mesmo membro com intervalo de dois minutos. A temperatura, frequência cardíaca e respiratória foi feita manualmente e comparada com os valores registrados no monitor. Em caso de divergencia de valores, foi realizada outra avaliação manual e feito a média das medidas manuais. Foram utilizados os piores valores dos parâmetros vitais e laboratoriais e suas pontuações foram calculadas a partir da calculadora online ${ }^{8}$.

As informações obtidas de cada paciente foram digitadas individualmente em um banco de dados organizados a partir de planilha eletrônica e estas foram confrontadas com as informações colhidas nos prontuários referentes ao tempo de permanência e taxa de mortalidade.

\section{Análise dos dados}

A amostra foi dada pela quantidade de pacientes admitidos na UTI do presente estudo, considerando os critérios de inclusão e exclusão. As variáveis qualitativas foram expressas em frequência absoluta e porcentagem e as variáveis quantitativas em média e desvio padrão. Os testes t de Student e o teste Mann Whitney foram usados para comparar as diferenças entre os grupos de sobreviventes e não sobreviventes, quanto à idade e o sexo. Para a variável sexo, foi utilizado o teste qui-quadrado com $p \leq 0,05$ para verificar o nível de significância Além disso, o coeficiente de correlação de Spearman foi usado para examinar a relação entre o escore APACHE-IV e o tempo de internação na UTI. Para verificar a acurácia do APACHEIV para mortalidade foi utilizado à curva Receiver
Operator Characteristic (ROC) com uma atribuição de 'bom'> 0,80. O teste de Mann Whitney foi utilizado para comparar a diferença entre os grupos sobrevivente e não sobrevivente. Para a variável sexo foi utilizado o teste do qui-quadrado com $p \leq 0,05$ para estatisticamente significativo. A análise dos dados foi feita pelo programa Stata ${ }^{\circledR}$.

\section{Resultados}

No período proposto para a pesquisa vinte (20) pacientes foram admitidos na UTI em estudo, entretanto, apenas dez (10) pacientes compuseram a amostra, pois, três (3) pacientes não apresentaram todos os exames necessários para avaliar o escore APACHE IV e os outros sete permaneceram internados na UTI após o término desta pesquisa (Figura 1).

A média da idade dos pacientes encontrados na UTI investigada foi de $44,80 \pm 22,59$ anos, sendo $70 \%$ dos pacientes do sexo masculino. $O$ tempo médio de permanência na UTI foi de 7,4 dias, com desvio padrão de $\pm 0,521$ (Tabela 1). Dos pacientes investigados seis apresentaram as seguintes alterações: neurológicas, endócrina, metabólica, respiratória, cardiovascular e renal, e quatro pacientes apresentaram alterações cardiovasculares e respiratórias.

Os resultados verificaram que houve uma diferença pouco significativa entre os dois grupos (sobreviventes e não sobreviventes) quanto ao sexo $(p=0,081)$. Entretanto, os dois grupos são significativamente diferentes em relação às variáveis: idade, tempo de permanência em UTI, RASS e APACHE IV, pois para todas essas variáveis o $p$ foi $<0,001$ (Tabela 1). O tempo de permanência na UTI previsto pelo APACHE IV foi de 14 dias $( \pm 7,4)$. Para a mortalidade geral os valores observados foram de $30 \%$, enquanto que a mortalidade prevista pelo APACHE foi de 50\% (Tabela 2).

A curva ROC para o escore APACHE IV e mortalidade observada foi descrita na Figura 2. Os valores encontrados foram estatisticamente significativos e podem ser um preditor para a mortalidade observada. No entanto, o coeficiente de correlação de Spearman apresentou correlação fraca entre o escore APACHE IV e o tempo de permanência em UTI observado ( $r=$ 0,$025 ; p<0,0001$ ). 
Figura 1. Fluxograma da amostra

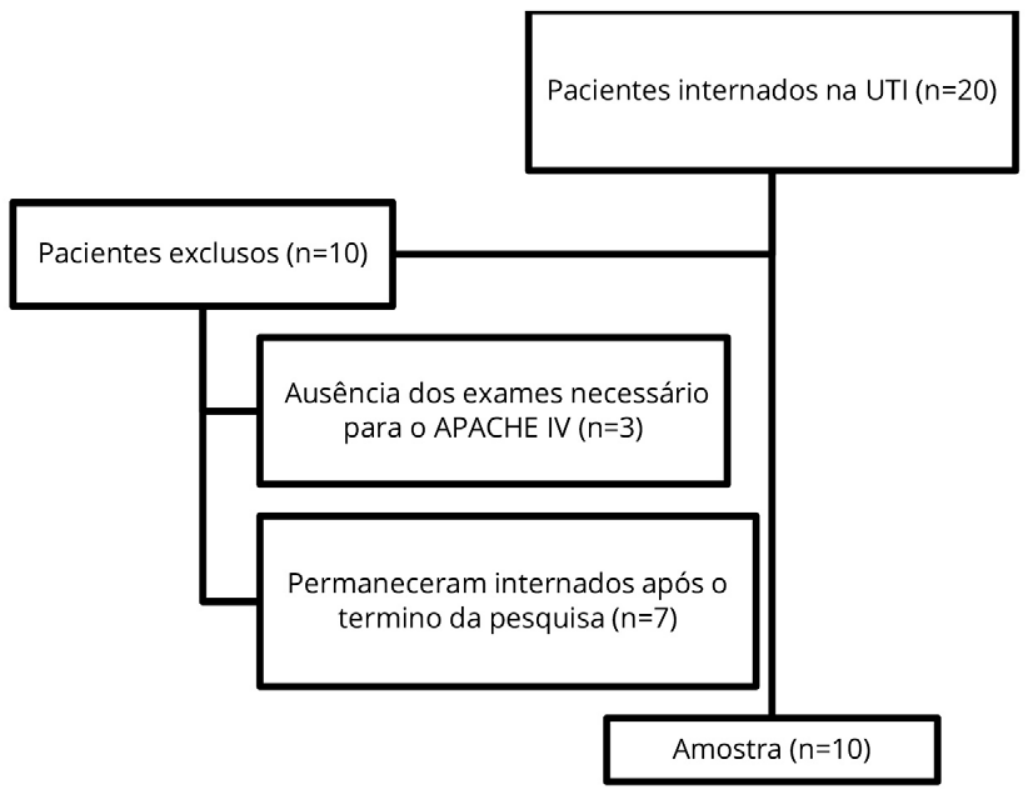

Tabela 1. Características demográficas e clínica dos pacientes internados na UTI do Hospital Regional de Gurupi em 2018

\begin{tabular}{llllr}
\hline Características & Total $(\mathrm{n}=10)$ & Sobreviventes $(\mathrm{n}=7)$ & Não sobreviventes $(\mathrm{n}=3)$ & $\mathrm{p}$-valor \\
\hline $\begin{array}{l}\text { Idade (média e dp) } \\
\text { Sexo (média e \%) }\end{array}$ & $44,80( \pm 22,59)$ & $37,71( \pm 8,97)$ & $61,33( \pm 2,72)$ & 0,000 \\
Masculino & $7(70 \%)$ & $5(71,43 \%)$ & $2(66,67 \%)$ & 0,081 \\
Feminino & $3(30 \%)$ & $2(28,57 \%)$ & $1(33,33 \%)$ & \\
UTI (Média e dp) & $7,4( \pm 0,52)$ & $7,71( \pm 0,52)$ & $6,67( \pm 1,33)$ & 0,000 \\
RASS (média e dp) & $-4,7( \pm 0,21)$ & $-4,71( \pm 0,28)$ & $-4,67( \pm 0,33)$ & 0,000 \\
APACHE IV (média e dp) & $75,70( \pm 6,67)$ & $70,0( \pm 3,34)$ & $89,0( \pm 21,54)$ & 0,000 \\
\hline
\end{tabular}

Tabela 2. Tempo de permanência e taxa de mortalidade encontrada e estimada pelo escore APACHE IV na UTI do Hospital Regional de Gurupi em 2018

\begin{tabular}{|c|c|c|c|}
\hline Característica & Observado & Previsto & Valor $\mathrm{p}$ \\
\hline
\end{tabular}




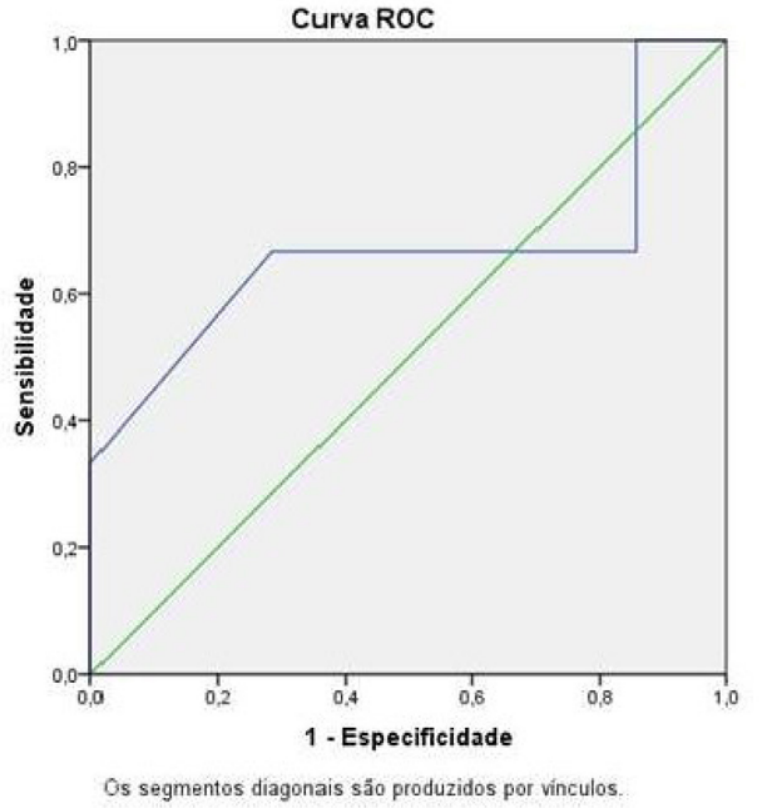

\section{Discussão}

Os resultados do presente estudo mostraram que o APACHE IV superestimou o período de permanência encontrado na UTI em estudo, com $(p<0,001)$. Além disso, mortalidade geral também foi superestimada com diferença absoluta de $20 \%(p=0,447)$.

A pesquisa em questão com dez pacientes não identificou o escore APACHE IV como bom preditor do tempo de permanência e da mortalidade em pacientes admitidos na UTI. Geralmente esses sistemas de pontuação funcionam adequadamente para a população em que foram testados. Especialistas recomendam a validação externa a nível nacional, regional ou institucional. Resultados semelhantes também foram encontrados em um estudo realizado no Irã com 839 pacientes, onde a taxa de mortalidade foi superior a prevista pelo escore APACHE IV7.

A utilização destes preditores de mortalidade em UTI vem sendo cada vez mais frequente, pois além de estimar o tempo de internação eles avaliam a gravidade da doença, auxiliando na conduta dos profissionais. Quanto menor o tempo que esses pacientes permanecerem internados em UTI, menores serão as chances de outras complicações ou até mesmo a piora da doença de base ${ }^{9}$.
O tempo médio estimado de permanência de pacientes em UTIs brasileiras é de seis dias, entretanto, alguns fatores podem prolongar essa internação, como por exemplo, a necessidade de assistência ventilatória ${ }^{10}$. Os doentes que necessitam de um prolongamento desta assistência estão expostos a uma série de complicações, entre elas a (PAVM) ${ }^{11}$. Dados semelhante encontrado na pesquisa, com tempo de permanência de $7,4 \pm 0,52$ dias.

A sedação é outro fator que interfere no tempo de permanência na UTI, pois quanto maior o nível de sedação, maior a necessidade de assistência ventilatória. A interrupção diária da sedação é uma estratégia para evitar a sedação excessiva, a fim de avaliar a necessidade de sedativo e diminuir o acúmulo, o tempo de ventilação mecânica e a permanência na UTI'2.

No presente estudo todos os pacientes estavam sob efeito de sedativos devido à gravidade no período da internação. A média encontrada da sedação avaliada pela escala de RASS nas primeiras 24 horas foi de $-4,7 \pm 0,21$. Entretanto, é rotina da UTI a interrupção diária da sedação, o que pode ter favorecido na redução do tempo de permanência se comparado com o previsto pelo APACHE IV. A maioria dos pacientes admitidos em UTIs é submetida a altas doses de sedativos, acarretando no aumento do tempo de internação, no prolongamento do uso do suporte ventilatório e no risco de óbito devido a outras possíveis complicações ${ }^{13}$. 
A média da idade dos pacientes encontrada em nosso estudo foi de $44,80 \pm 22,59$ anos. Alguns estudos afirmam que a idade avançada está associada ao prolongamento no tempo de internação e a altas taxas de mortalidade ${ }^{14}$. Dados que corroboram com esta pesquisa evidenciou que os sobreviventes tinham uma média de idade baixa $(37,71 \pm 8,97)$ quando comparado aos não sobreviventes $(61,33 \pm 2,72)$.

A confiabilidade do APACHE IV tem sido questionada e comparada com outros preditores de mortalidade e tempo de permanência. Alguns estudos verificaram a ineficácia do escore APACHE IV para estimar o tempo de permanência, por falta de boa calibração. Em contrapartida, eles observaram uma boa predição para o tempo de mortalidade. Outros autores divergem desta opinião e caracterizaram o APACHE IV como um bom preditor de mortalidade e permanência ${ }^{15}$.

Um estudo realizado na República Checa avaliou 1.000 pacientes, com média de idade de 69,9 anos, sendo a maior porcentagem do sexo masculino $(n=560)$, acometimentos por doenças cardiopulmonares, respiratórias e/ou gastrointestinais. Este comparou a confiabilidade dos escores APACHE II, APACHE IV, SAPS 3 e MPMo III, e pode observar que o APACHE IV obteve resultado estimado mais próximo do encontrado para o tempo de permanência dos pacientes avaliados ${ }^{16}$.

Outras duas pesquisas que equipararam os escores: APACHE II, APACHE IV e SAPS 3 constataram que o APACHE IV revelou uma boa discriminação, mas uma baixa calibração quanto ao tempo de permanência dos pacientes em UTI. No primeiro estudo, no período de janeiro a de junho de 2014, foram avaliados 1.003 pacientes, sendo $65 \%$ do sexo masculino e 70\% admitidos por acometimentos cardíacos, respiratórios e metabólicos e os outros $30 \%$ por problemas cirúrgicos. No segundo estudo 1.314 pacientes com média de idade de 57,8 anos, sendo $57 \%$ do sexo masculino compuseram a amostra. Destes pacientes 516 passaram por neurocirurgia. Em ambas pesquisas utilizaram os piores valores durante as primeiras 24 horas de internação para calcular o escore APACHE IV ${ }^{17-18}$. Entretanto, no presente estudo observou-se, uma correlação fraca entre o escore APACHE IV e o tempo de permanência na UTI investigada $(r=0,025 ; p<0,0001)$.
Embora o estudo traga importantes contribuições, apresentou limitação no tamanho da amostra em função do número reduzido de leitos, baixa rotatividade e tempo insuficiente de acompanhamento dos pacientes admitidos na UTI.

\section{Conclusão}

O escore APACHE IV não demonstrou confiabilidade para predição de mortalidade e tempo de permanência na UTI investigada. Recomenda-se estudos com maior população, com caracterísitcas demográficas semelhantes à população brasileira e durante um maior tempo de acompanhamento.

\section{Contribuições dos autores}

Carvalho GA participou da concepção, da coleta de dados da pesquisa, interpretação dos resultados, redação do artigo científico. Rezende AAB participou da concepção, delineamento, busca e análise estatística dos dados da pesquisa, interpretação dos dados e redação do artigo científico. Reis GR participou da concepção, da coleta de dados da pesquisa e da interpretação dos resultados. Gardenghi $\mathrm{G}$ participou da interpretação dos resultados e da redação do artigo científico.

\section{Conflitos de interesses}

Nenhum conflito financeiro, legal ou político envolvendo terceiros (governo, empresas e fundações privadas, etc.) foi declarado para nenhum aspecto do trabalho submetido (incluindo, mas não se limitando a subvenções e financiamentos, participação em conselho consultivo, desenho de estudo, preparação de manuscrito, análise estatística, etc.).

\section{Referências}

1. Roque KE, Tonini T, Melo ECP. Eventos adversos na unidade de terapia intensiva: impacto na mortalidade e no tempo de internação em um estudo prospectivo. Cad. Saúde Pública. 2016;32(10). doi: 10.1590/0102-311X00081815

2. Mota EC, Oliveira SP, Silveira BRM, Silva PLN, Oliveira AC. Incidência da pneumonia associada àventilação mecânica em unidade de terapia intensiva. Medicina (Ribeirão Preto, Online). 2017;50(1):39-46. doi: 10.11606/issn.2176-7262.v50i1p39-46 
3. Feijó CAR, Leite Júnior FO, Martins ACS, Furtado Júnior AH, Cruz LLS, Meneses FA. Gravidade dos pacientes à unidade de terapia intensiva de um hospital universitário Brasileiro. Rev Bras Ter Intensiva. 2006;18(1):18-21. doi: 10.1590/S0103507X2006000100004

4. Keegan MT, Gajic O, Afessa B. Comparison of APACHE IV Resuscitation Status on Model Performance. Chest. 2012;142(4):851-8. doi: 10.1378/chest.11-2164

5. Brinkman S, Bakhshi-Raiez F, Abu-Hanna A, Jonge E, Bosman RJ, Peelen L et al. External validation of Acute Physiology and Chronic Health Evaluation IV in Dutch intensive care units and comparison with Acute Physiology and Chronic Health Avaluation II and Simplified Physiology Score II. J Crit Care. 2011;26(1):105e11-8. doi: 10.1016/j.jerc.2010.07.007

6. Rodriguez AH, Bub MBC, Perão OF, Zandonadi G, Rodriguez $\mathrm{MJH}$. Características epidemiológicas e causas de óbitos em pacientes internados em terapia intensiva. Rev Bras Enferm. 2016;69(2):229-34. doi: 10.1590/0034-7167.2016690204i

7. Ghorbani M, Ghaem H, Rezaianzadeh A, Shayan Z, Zand F, Nikandish R. A study on the efficacy of APACHE- IV for predicting mortality and length of stay in na intensive care unit in Iran. F1000 Res. 2017;6:2032. doi: 10.12688/f1000research.12290.1

8. Calculadoras ICU- RNSH. APACHE IV pontuação. [Internet]. [Acesso em 2018 out. 05]. Disponível em: https:// intensivecarenetwork.com/Calculators/Files/Apache4.html

9. Keegan MT, Soares M. O que todo intensivista deveria saber sobre os sistemas de escore prognósticos de mortalidade ajustada ao risco. Rev Bras Ter Intensiva. 2016;28(3):264-9. doi: 10.5935/0103-507X.20160052

10. Orlando JMC, Milani CJ. $2^{\circ}$ Anuário Brasileiro de UTIs - $2^{\circ}$ Censo Brasileiro de UTIs. São Paulo: Associação de Medicina Intensiva Brasileira (AMIB); Edição 2002-2003.

11. Mota EC, Oliveira SP, Silveira BRM, Silva PLN, Oliveira AC. Incidência da pneumonia associada à ventilação mecânica em unidade de terapia intensiva. Medicina (Ribeirão Preto, online). 2017;50(1):39-46. doi: 10.11606/issn.2176-7262.v50i1p39-46

12. Carvalho CRR, Toufen Junior $C$, Franca SA. III Consenso Brasileiro de Ventilação Mecânica. J Bras Pneumol. 2007;33(supl 2):54-70.

13. Shinotsuka CR, Salluh JIF. Percepções e práticas sobre delirium, sedação e analgesia em pacientes críticos: uma revisão narrativa. Rev Bras Ter Intensiva. 2013;25(2):155-61. doi: 10.5935/0103-507X.20130027.

14. Williams TA, Ho KM, Dobb GJ, Finn JC, Knuiman M, Webb SA et al. Effect of length of stay in intensive care unit on hospital and long-term mortality of critically ill adult patients. $\mathrm{Br} J$ Anaesth. 2010;104(4):459-64. doi: 10.1093/bja/aeq025
15. Choi JW, Park YS, Lee YS, Park YH, Chung C, Park DI et al. The Ability of the Acute Physiology and Chronic Health Evaluation (APACHE) IV Score to Predict Mortality in a Single Tertiary Hospital. Korean J Crit Care Med. 2017;32(3):275-83. doi: 10.4266/ kjccm.2016.00990

16. Sedlon P, Kameník L, Skvarill J, Malý M, Táborský M, Zavoral M. Comparison of the accuracy and correctness of mortality estimates for Intensive Care Unit patients in internal clinics of the Czech Republic insing APACHE II, APACHE IV, Saps 3 and MPMo III models. Med Glas. 2016;13(2):82-9. doi: 10.17392/860-16

17. Varghese YE, Kalaiselva MS, Renuka MK, Arunkumar AS. Comparison of acute physiology and chronic health evaluation II (APACHE II) and acute physiology and chronic health evaluation IV (APACHE IV) severity of illness scoring systems, in a multidisciplinary ICU. J Anaesthesiol Clin Pharmacol. 2017;33(2):248-53. doi: 10.4103/0970-9185.209741

18. Lee $H$, Shon YJ, Kim H, Paik H, Park HP. Validation of the APACHE IV model and its comparison with the APACHE II, SAPS 3, and Korean SAPS 3 models for the prediction of hospital mortality in a Korean surgical intensive care unit. Korean J Anesthesiol. 2014;67(2):115-22. doi: 10.4097/kjae.2014.67.2.115 